\title{
An apoptosis signaling pathway induced by the death domain of FADD selectively kills normal but not cancerous prostate epithelial cells
}

\author{
MJ Morgan ${ }^{1,4}$, J Thorburn ${ }^{2,4}$, L Thomas ${ }^{2}$, T Maxwell $^{3}$, \\ AR Brothman ${ }^{3}$ and A Thorburn ${ }^{\star 2}$ \\ ${ }^{1}$ Huntsman Cancer Institute, Department of Oncological Sciences, University of \\ Utah, Salt Lake City, Utah 84112 USA \\ 2 Department of Cancer Biology, Wake Forest University School of Medicine, \\ Winston-Salem, North Carolina 27157, USA \\ ${ }^{3}$ Departments of Pediatrics and Human Genetics, University of Utah, Salt Lake \\ City, Utah 84112, USA \\ ${ }^{4}$ These authors contributed equally. \\ * Corresponding author: Andrew Thorburn, Wake Forest University School of \\ Medicine, Department of Cancer Biology, Medical Center Boulevard, Winston- \\ Salem, NC. 27157, USA. Tel. 336-716-7587; Fax. 336-716-0255; \\ E-mail: athorbur@wfubmc.edu
}

Received 23.11.00; revised 6.2.01; accepted 20.2.01

Edited by G Nunez

\begin{abstract}
The adaptor protein FADD directly, or indirectly via another adaptor called TRADD, recruits caspase 8 to death receptors of the tumor necrosis factor receptor family. Consequentially, a dominant-negative mutant (FADD-DN, which consists only of the FADD death domain) that binds to receptors but cannot recruit caspase 8 has been widely used to inhibit apoptosis by various stimuli that work via death receptors. Here, we show that FADD-DN also has another cell type- and cancerdependent activity because it induces apoptosis of normal human prostate epithelial cells but not normal prostate stromal cells or prostate cancer cells. This activity is independent of FADD-DN's ability to bind to three known interacting proteins, Fas, TRADD or RIP suggesting that it is distinct from FADD's functions at activated death receptors. FADD-DN induces caspase activation in normal epithelial cells as demonstrated using a Fluorescence Resonance Energy Transfer assay that measures caspase activity in individual living cells. However, caspase-independent pathways are also implicated in FADD-DN-induced apoptosis because caspase inhibitors were inefficient at preventing prostate cell death. Therefore, the death domain of FADD has a previously unrecognized role in cell survival that is epithelial-specific and defective in cancer cells. This FADD-dependent signaling pathway may be important in prostate carcinogenesis. Cell Death and Differentiation (2001) 8, 696-705.
\end{abstract}

Keywords: FADD; prostate cancer; epithelia; FRET

Abbreviations: AIF, apoptosis inducing factor; BHA, butylated hydroxyanisole, BFP, blue fluorescent protein; ERK, extra- cellular signal-regulated protein kinase; DISC, death-inducing signaling complex; FADD, Fas associated protein with a death domain; FADD-DN, dominant negative FADD; FRET, fluorescence resonance energy transfer; MAP kinase, mitogenactivated protein kinase, MEK, MAP kinase kinase; TNF, tumor necrosis factor: TRAIL, TNF-related apoptosis inducing ligand

\section{Introduction}

Prostate cancer is the most commonly diagnosed non-skin malignancy in men and the second leading cause of male cancer-related deaths accounting for approximately 32000 deaths in the United States in the year 2000. ${ }^{1}$ Like other cancers, prostate cancer is associated with defective cellular responses to apoptotic stimuli and a hallmark of cancer cells is that they must possess defects in signaling pathways that regulate apoptosis. ${ }^{2}$ However, despite important exceptions such as the overexpression of anti-apoptotic Bcl-2 proteins, it is largely unclear exactly what apoptosis signaling pathways are defective in cancer cells compared to their normal counterparts. The best understood signaling pathways that regulate apoptosis are those induced by activated death receptors such as Fas. ${ }^{3}$ Normal prostate epithelium expresses both Fas and its ligand and several studies implicate a role for Fas signaling in prostate cancer ${ }^{4-6}$ and during prostate cell apoptosis after androgen removal. ${ }^{7,8}$

Ligand binding to death receptors such as Fas leads to the formation of the Death Inducing Signaling Complex (DISC) that recruits adaptor proteins including Fas Associated Death Domain protein (FADD) and initiator caspases such as caspase 8 . Aggregation of caspase 8 in the DISC leads to the activation of a caspase cascade and apoptosis. FADD consists of two protein interaction domains, a death domain and a death effector domain. ${ }^{9}$ Because FADD is an essential component of the DISC, a dominant negative mutant (FADD-DN) that contains the death domain but no death effector domain has been widely used in studies of death receptor-induced apoptosis. FADD-DN functions as a dominant negative inhibitor because it binds to the receptor but cannot recruit caspase 8 and has been used to determine whether FADD is required for diverse apoptotic stimuli. ${ }^{10-21}$ Here, we show that FADD-DN also has an unexpected and quite different activity in that it induces caspase activation and apoptosis in normal human prostate epithelial cells. This novel activity is distinct from FADD's role in the Fas pathway and does not occur in non-epithelial cells or prostate tumor cells. These characteristics imply that this FADD death domain-dependent activity may be involved in prostate carcinogenesis. 


\section{Results \\ FADD-DN induces apoptosis in normal prostate epithelial cells}

To investigate FADD signaling in prostate cells, a FADD-DN expression plasmid encoding amino acids 80-208 of FADD (the death domain) with a yellow fluorescent protein (YFP) molecule in place of the death effector domain was injected into primary cultures of normal human luminal prostate epithelial cells. Direct plasmid injection leads to rapid expression of reporters such as $\mathrm{YFP}^{22}$ and successfully injected cells were identified within $2 \mathrm{~h}$ by virtue of their fluorescence. YFP-FADD-DN- and control YFP-injected cells were monitored over time. Surprisingly, we found that the FADD-DN-injected cells rounded up and died within a few hours. Figure 1a shows the results of quantitative cell survival assays from experiments performed with three separate primary cell preparations. Death was dependent upon expression of the YFP-FADD-DN fusion protein since control injections with the YFP expression plasmid did not kill the cells. Furthermore, the effect was specific to the FADD death domain because YFP fused to the death domain of DAP kinase, a molecule that is downstream of FADD in the Fas pathway, ${ }^{23}$ did not kill the cells. As expected for a dominant-interfering mutant, the response to FADD-DN was dose-dependent such that injection of increasing concentrations of the expression plasmid were more effective at inducing cell death (Figure 1b). Time-lapse fluorescence microscopy demonstrated that FADD-DN expressing cells contract, round up, detach from the plate and produce intact membrane blebs containing the fluorescent marker (Figure 1c). These characteristics suggest that this death is apoptotic rather than necrosis, which leads to cell swelling and membrane lysis.

When FADD-DN injected cells were fixed before detachment and stained to allow visualization of nuclei, we found that the nuclei of the dying cells were condensed consistent with apoptosis (Figure 2a). Apoptotic cells display changes in their cell membranes such that phosphatidylserine is translocated from the inner to the outer membrane, which can be detected by staining non-permeabilized cells with the phosphatidylserine-binding protein annexin V. FADD-DNinjected cells but not control-injected cells stained with annexin $V$ (Figure 2b) indicating that translocation of phosphatidylserine occurs in response to FADD-DN injection. We also fixed and stained injected cells with an antibody (M30) that detects caspase-cleaved cytokeratin 18. Figure $2 \mathrm{c}$ shows that FADD-DNinjected, but not YFP-injected cells were stained with this antibody suggesting that caspases are active in the injected cells. FADD-DN injection into normal prostate epithelial cells is therefore associated with cell condensation, membrane blebbing, nuclear condensation, phosphatidylserine translocation to the outer membrane and the appearance of a caspase-dependent epitope indicating that FADD-DN induces apoptosis in these cells.

\section{FADD-DN induces apoptosis only in normal epithelial cells}

The fact that FADD-DN should induce prostate cell apoptosis is surprising because the mutant is a well-characterized inhibitor of apoptosis in numerous cell types that have been tested. To determine whether FADD-DN-induced death was specific to the normal prostate epithelial cells, we injected the expression plasmids into a further ten different primary preparations of normal prostate epithelial cells. In addition, we tested two prostate tumor cell lines (PPC1, a line derived from a primary tumor and $\mathrm{LNCaP}$, a line derived from a metastatic lesion ${ }^{24,25}$ ), and normal human skin fibroblasts. FADD-DN killed only the normal prostate epithelial cells (Figure 3a). Four other prostate cell lines (CA HPV-10, PZHPV-7, PC3 and DU145) were also resistant to FADD-DN injection as were several non-prostate cell lines including Hela, NIH3T3 and HEK293 cells (data not shown). We then compared the ability of FADD-DN to kill normal prostate epithelial, fibroblast and smooth muscle cells. Only epithelial cells were killed by FADD-DN (Figure 3b). To test whether primary tumor cells as opposed to tumor-derived cell lines were killed by FADD-DN, we cultured epithelial cells from tissue pieces that contained tumor or from matched nontumor tissue from the same patient and compared cell survival after injection of the YFP control or YFP-FADD-DN. Figure 3c shows data from three patients indicating that only the FADDDN-injected epithelial cells from normal tissue showed significant cell death. Therefore, in the prostate, only noncancerous epithelial cells are sensitive to FADD-DN-induced apoptosis.

\section{FADD-DN induces caspase activation in normal but not cancerous prostate cells}

Most apoptosis is associated with caspase activation and the FADD-DN injected cells stained with an antibody that recognizes a caspase-cleaved protein, we therefore wished to test whether caspases were activated in FADD-DN-injected normal prostate epithelial cells. Because we can only inject a small number of primary prostate cells, conventional biochemical assays for caspase activity were impractical. We therefore used a new Fluorescence Resonance Energy Transfer (FRET)-based caspase assay that can continuously monitor caspase activity in individual, living cells. ${ }^{26}$ This assay uses a YFP-BFP fusion protein that is cleaved by caspase 3 (and other effector caspases that recognize DEVD motifs). The intact protein fluoresces yellow (because of FRET between BFP and YFP) upon excitation of the BFP protein with UV-light. Upon cleavage with caspase, yellow fluorescence decreases with a concomitant increase in blue fluorescence caused by conventional excitation and emission of BFP. The ratio of yellow/blue fluorescence in cells expressing the fusion protein is a measure of the intact/ cleaved protein and thus the caspase activity.

Normal prostate cells were injected with an expression vector to produce the YFP-BFP protein along with a FADDDN expression plasmid that lacked the YFP tag, or the corresponding control plasmid. After injection, the cells were maintained in an environmental chamber on the 



C. FADD-DN
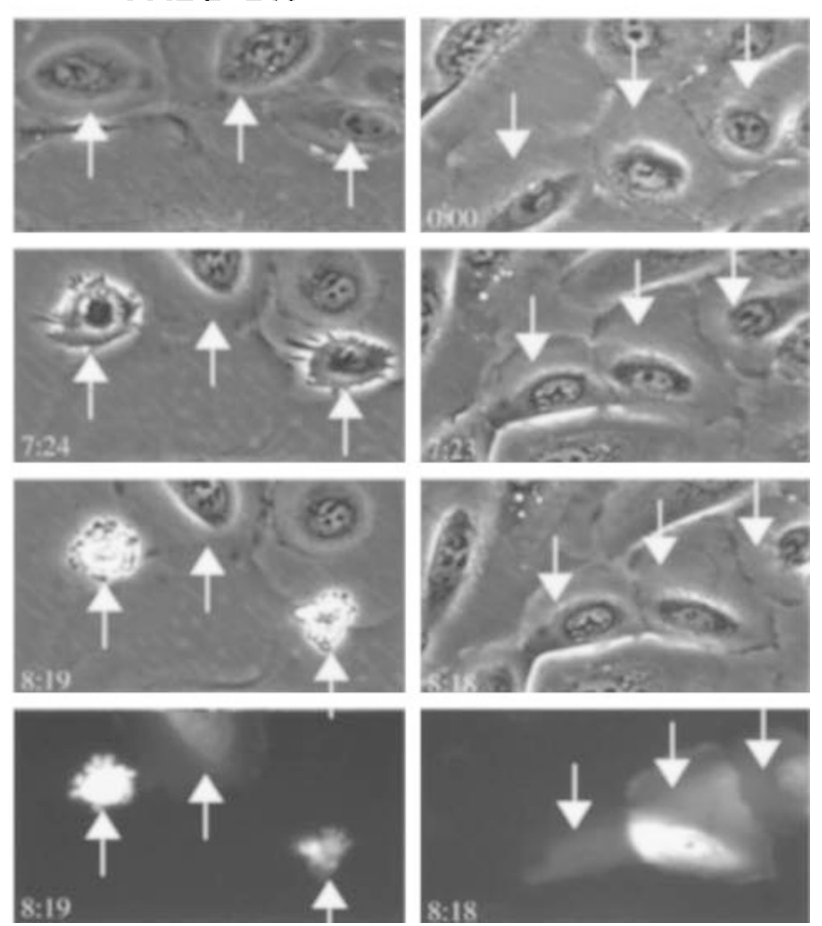

Figure 1 FADD-DN kills normal human prostate cells. (a) Normal human prostate epithelial cells were injected with an expression plasmid encoding

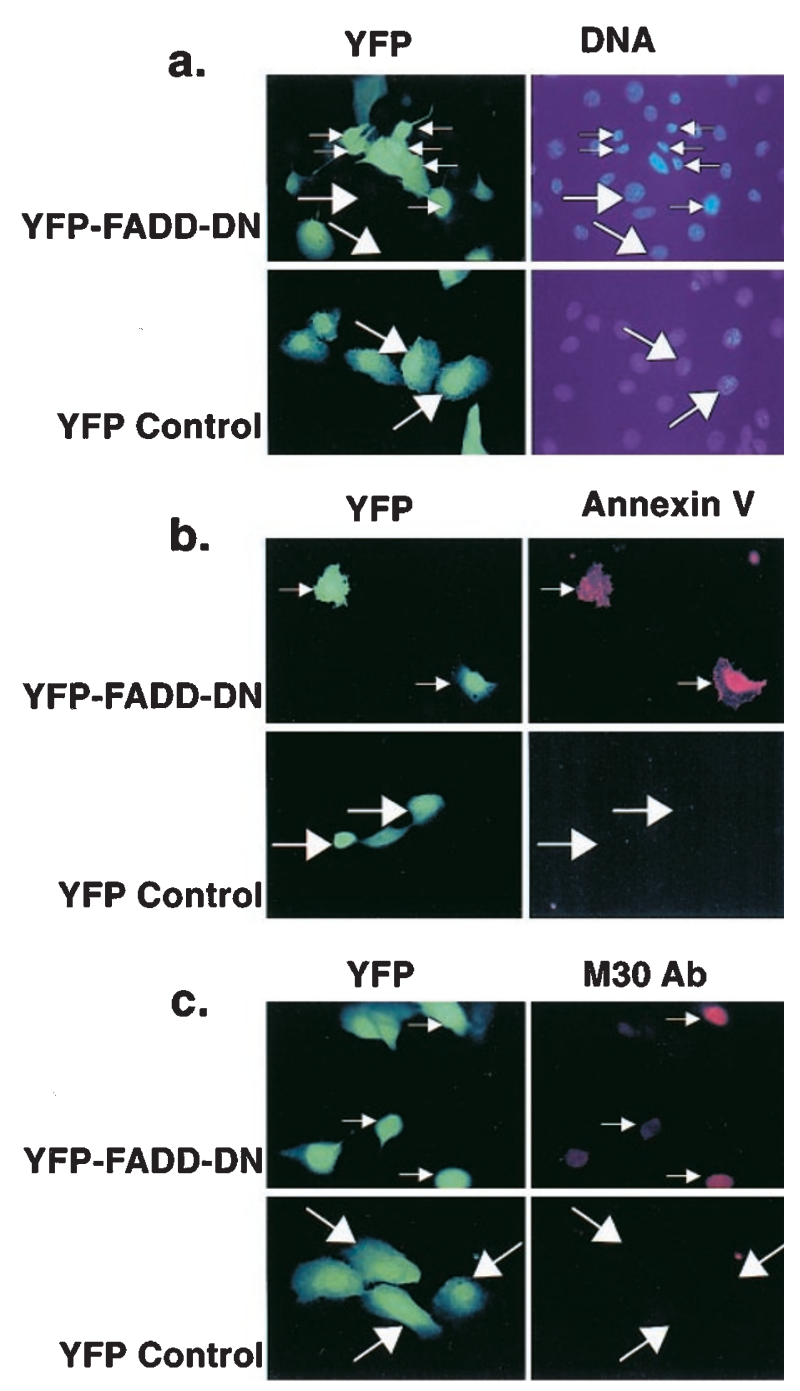

Figure 2 FADD-DN induces apoptosis of normal prostate epithelial cells. Cells were injected with YFP or YFP-FADD-DN expression plasmids. (a) A DNA stain indicating that FADD-DN-injected cells have condensed chromatin typical of apoptotic cells. (b) Staining of unfixed cells with Texas Redconjugated annexin $\mathrm{V}$, indicating that FADD-DN injected cells have phosphatidylserine on their outer membrane. (c) Staining with the M30 antibody, which detects caspase-cleaved cytokeratin 18 . Small arrows identify typical FADD-DN-injected cells large arrows identify typical control-injected or uninjected cells

YFP, YFP-FADD-DN or YFP fused to the death domain of DAP kinase. The per cent survival after overnight incubation was determined. The bars represent the mean \pm S.E.M. from three separate experiments $(50-100$ cells per sample for each experiment) using different preparations of primary cells. (b) The indicated amounts of the YFP-FADD-DN plasmid were injected into normal prostate cells. The total injected DNA was made up to $250 \mathrm{ng} / \mathrm{ml}$ using the empty YFP expression plasmid. Survival data is presented as the mean \pm S.E.M. for three separate preparations of normal prostate cells; the number above each bar indicates the total number of injected cells that were monitored. (c) Time-lapse images of the same field were captured at the indicated times after injection of cells with untagged FADD-DN expression plasmids or the corresponding vector and an expression plasmid that encodes YFP. Arrows indicate the injected cells, which were identified by the presence of the fluorescent marker. Control cells and uninjected cells remained flat whereas two of the three FADD-DN-injected cells rounded up, blebbed and died. The fluorescence image shows that these cells and their membrane blebs retain the fluorescent marker indicating that cell membranes remain intact. The third, FADD-DN-injected cell died several hours later (not shown) 

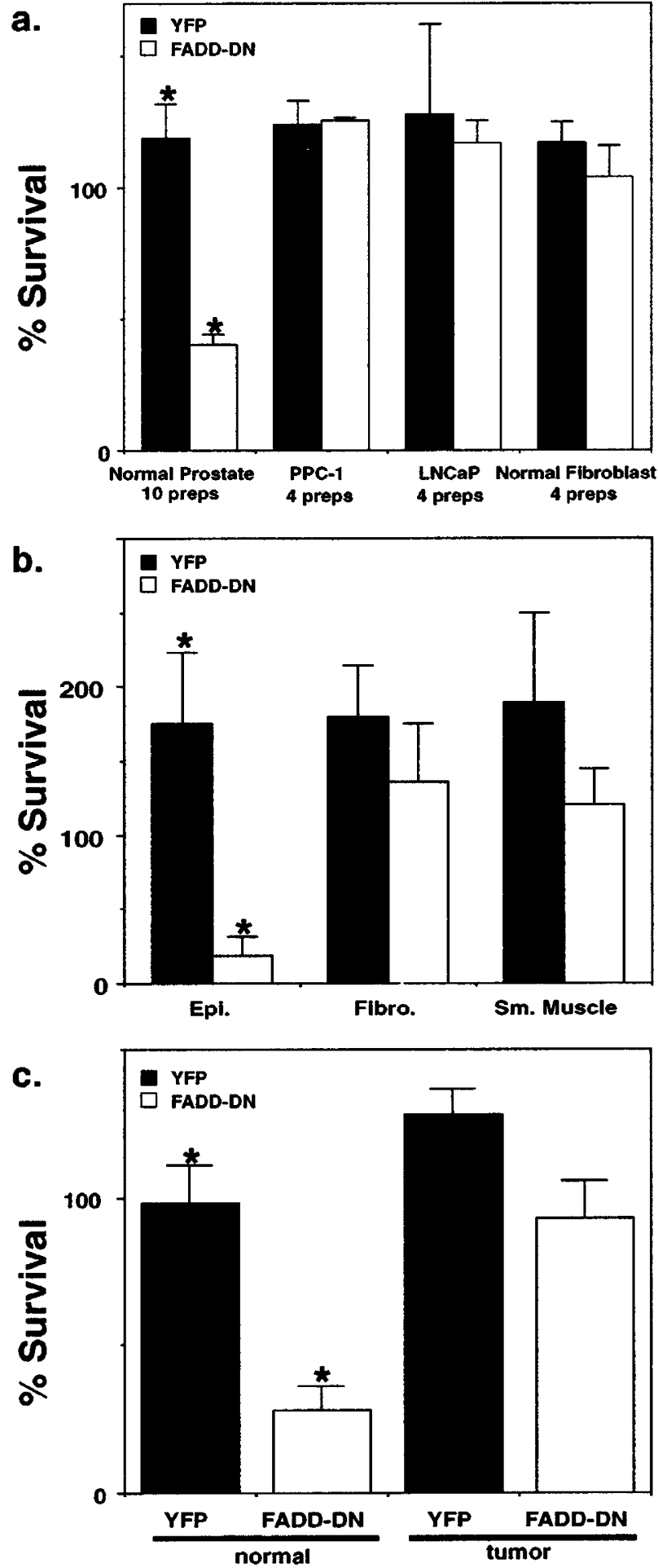

Figure 3 FADD-DN selectively kills normal prostate epithelial cells. (a) Survival data from 10 different preparations of normal prostate cells and four preparations each for PPC1 and LNCaP prostate cancer cells and normal human skin fibroblasts. Cells were injected with the control YFP vector or the YFP-FADD-DN expression plasmid. (b) Primary human prostate epithelial cells, prostate fibroblasts or smooth muscle cells were injected with YFP or YFP-FADD-DN expression plasmid. Cell survival data is presented from five experiments. Only the normal epithelial cells showed a statistically significant difference between control- and YFP-FADD-DN injected cells indicating that they are killed by FADD-DN. (c) Epithelial cells were cultured from matched microscope and yellow, blue and phase images were captured for $18 \mathrm{~h}$ after excitation with UV. Figure $4 \mathrm{a}$ shows pseudocolored images of the yellow/blue ratio and phase images for two FADD-DN injected cells at various time points, indicating that the yellow/blue fluorescence ratio of the injected cells decreases over time. Figure $4 \mathrm{~b}$ shows quantitative data from several FADD-DN-injected cells over time. For each cell, the yellow/blue fluorescence ratio decreases in a time-dependent manner prior to cell death indicating that the YFP-BFP fusion protein was cleaved in these cells.

a.

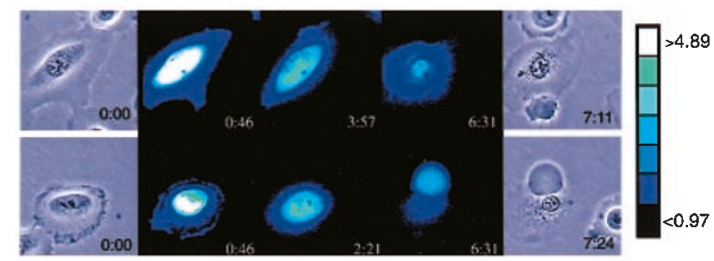

b.



c.

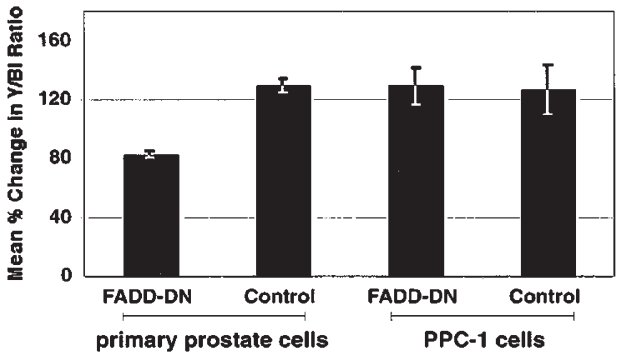

Figure 4 Caspase activation in FADD-DN-injected prostate cells. Normal prostate cells injected with an expression plasmid encoding the caspasecleavable YFP-BFP fusion protein and an untagged FADD-DN molecule. Yellow (FRET) and blue (conventional) fluorescence after excitation at $380 \mathrm{~nm}$ were monitored over time and the yellow/blue fluorescence ratio was calculated for each cell at various times. (a) Phase and pseudocolored yellow/blue ratios for two FADD-DN-injected cells at various times. The ratio of yellow to blue fluorescence decreases over time. (b) Quantitation of the ratio for five individual FADD-DN-injected cells over time indicating time-dependent cleavage of the fusion protein. (c) The mean change in ratio for groups of control and FADD-DN injected normal cells or PPC1 cells. These means for control- and FADD-DN-injected normal cells are different with a significance level $P<0.0001$ by $t$-test. The means for PPC 1 cells are not different $(P=0.46)$. These data indicate that FADD-DN expression causes caspase activation in normal prostate epithelial cells but not PPC1 cells

tissue pieces containing tumor tissue or normal tissue from three individuals. Normal and tumor cells were injected with YFP or YFP-FADD-DN. Only the FADD-DN-injected normal cells were killed. Each histogram represents the mean per cent survival \pm S.E.M. ${ }^{*}$ Indicates that the means are different $(P<0.01$ by $t$-test) none of the other pairs reached statistical significance 
To test whether caspases were differentially activated in control and FADD-DN injected cells we measured the relative change in yellow/blue ratio. The per cent change 8-10 h after injection (i.e. several hours before cell death occurred) for a group of either FADD-DN or control cells was determined for both normal prostate epithelial cells and PPC1 tumor cells (Figure 4c). For both cell types, the control plasmid caused an increase in the mean yellow/ blue ratio. This increase was due to continued expression of the fusion protein during the experiment. When FADDDN-injected cells were analyzed, the normal cells showed a decrease in the yellow/blue ratio while the PPC1 cells were similar to the control-injected cells. The difference between the means for the control compared to FADD-DN cells was highly significant $(P<0.0001$ by $t$-test) for the normal cells but was not different $(P=0.46)$ for the PPC1 tumor cell line. These data indicate that the normal cells activate caspases that can cleave the YFP-BFP fusion protein after FADD-DN injection but that the PPC1 cells do not. Therefore, in normal human prostate epithelial cells, the isolated death domain of FADD activates caspases via a mechanism that is distinct from aggregation of caspase 8 such as occurs at the DISC. This does not occur in prostate tumor cells, which do not display any activation of caspases and do not die in response to FADD-DN.

\section{Caspase inhibitors, antioxidants and $\mathrm{Bcl}-\mathrm{x}_{\mathrm{L}}$ do not efficiently prevent FADD-DN-Induced death}

To determine whether caspase activation in response to FADD-DN injection was required for prostate cell apoptosis, we treated cells with the caspase inhibitor zVAD.fmk. Figure $5 \mathrm{a}$ shows that even at high concentrations $(500 \mu \mathrm{M})$, the inhibitor could only very slightly inhibit FADD-DN-induced cell death. This effect was much less than that observed in control experiments where we overexpressed full-length FADD (containing both the death domain and death effector domain) in Hela cells and obtained complete protection against apoptosis (data not shown). These data suggest that caspase-independent signals play a role in FADD-DN-induced apoptosis of normal prostate epithelial cells. Co-expression of the baculovirus p35 protein was also only modestly effective at preventing FADD-DN-induced death of prostate epithelial cells (data not shown).

Recent reports indicate that other death domains for example from TNFR1 can kill cells via necrosis that can be inhibited by antioxidants such as $100 \mu \mathrm{M}$ butylated hydroxyanisole $(\mathrm{BHA})^{27}$ and this can be stimulated zVAD.fmk. Figure $5 \mathrm{~b}$ shows that addition of $\mathrm{BHA}$ along with $150 \mu \mathrm{M}$ zVAD.fmk did not prevent FADD-DN-induced death. These data suggest that the cell death mechanisms that are activated by FADD-DN in normal prostate cells are different from those induced by the TNFR1 death domain. ${ }^{27}$ We also tested whether the anti-apoptotic $\mathrm{Bcl}-\mathrm{x}_{\mathrm{L}}$ protein could prevent FADD-DN-induced death. Co-injection of a $\mathrm{BCl}-\mathrm{x}_{\mathrm{L}}$ expression plasmid along with the FADD-DN expression plasmid did not significantly increase cell survival (Figure 5c).

\section{FADD-DN-induced prostate cell apoptosis is not caused by binding to Fas, TRADD or RIP}

FADD-DN consists only of a protein interaction domain and blocks Fas-induced apoptosis because it can interact with the death domain of the Fas receptor. ${ }^{3}$ We therefore wished to determine whether interaction with Fas or other known binding partners was required for FADD-DN-induced death of normal prostate cells. We constructed several FADD-DN mutants and determined their ability to interact in directed two-hybrids with the death domains of Fas or two other DISC proteins (TRADD and RIP) that interact with FADD. ${ }^{11,28}$ The mutants showed variable abilities to induce prostate cell apoptosis (Figure 6a) and also differed in their ability to interact with Fas, TRADD and RIP (Figure 6b, c and d). A summary of these data is shown in Figure $6 e$. There was no correlation between binding to any of these known FADD targets and the ability to induce prostate epithelial cell apoptosis. For example, neither the C168 stop or the V121N mutants bound efficiently to TRADD yet both mutants were equally effective as the wild-type FADD-DN at inducing prostate cell apoptosis. Conversely, the A174P mutant, which binds equally well as the wild-type to TRADD was inhibited in its ability to induce apoptosis. There was also no correlation between RIP binding and ability to induce apoptosis. For example, both V121N and A174P mutants bound RIP somewhat better than the wild-type FADD-DN yet differ in their ability to induce apoptosis. A similar lack of correlation was also found for Fas binding. The C168 stop mutant showed significantly reduced binding to Fas but was equally potent as the wild-type FADD-DN at inducing prostate cell apoptosis. Further data supporting a lack of correlation between Fas binding and ability to induce apoptosis was provided by the D175A mutant which was compromized in its ability to kill cells but bound Fas as well as the wild-type protein. Taken together, these data suggest that the ability of FADD-DN to induce prostate cell apoptosis is not mediated through interaction with any of these three known interacting proteins that are components of the DISC.

\section{Discussion}

We show that FADD-DN, a widely used inhibitor of apoptosis induced by death receptors of the TNF family, ${ }^{3,13}$ has a novel and unexpected effect in normal human prostate epithelial cells where it causes caspase activation and apoptosis. FADD-DN is unable to kill primary prostate tumor cells, prostate cancer cell lines, normal prostate fibroblasts and smooth muscle cells or normal skin fibroblasts. Thus, the effect is cell type-specific and defective in prostate cancer cells. Numerous apoptosis signaling proteins have been characterized to date. However, there are few examples where a mutant signaling protein displays a cell type-specific ability to induce apoptosis in normal human cells that is defective in corresponding tumor cells from the same surgical specimen. This difference in responsiveness between normal cells and tumor cells from the same tissue implies that the signaling pathway that is affected by FADD-DN may be directly involved in carcinogenesis. 


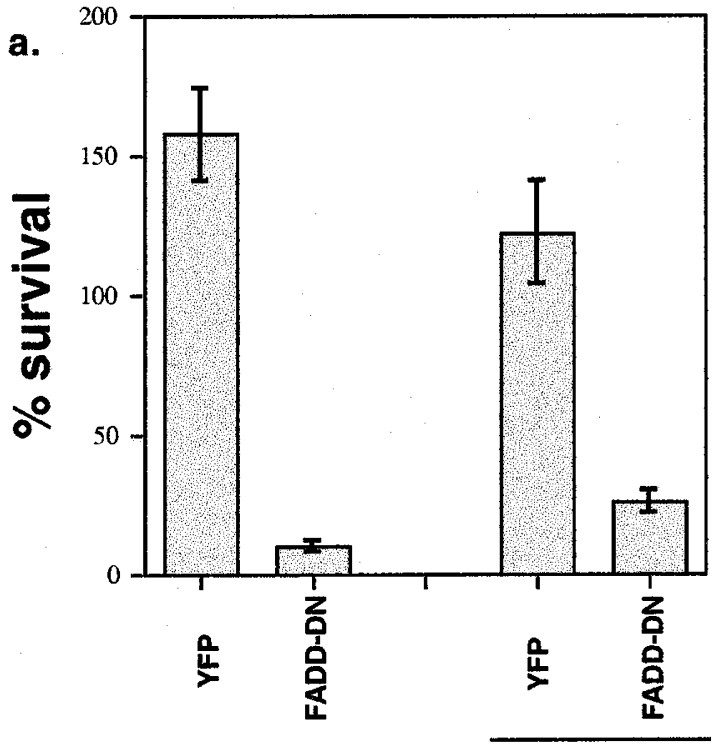

$500 \mu M$ zVAD.fmk
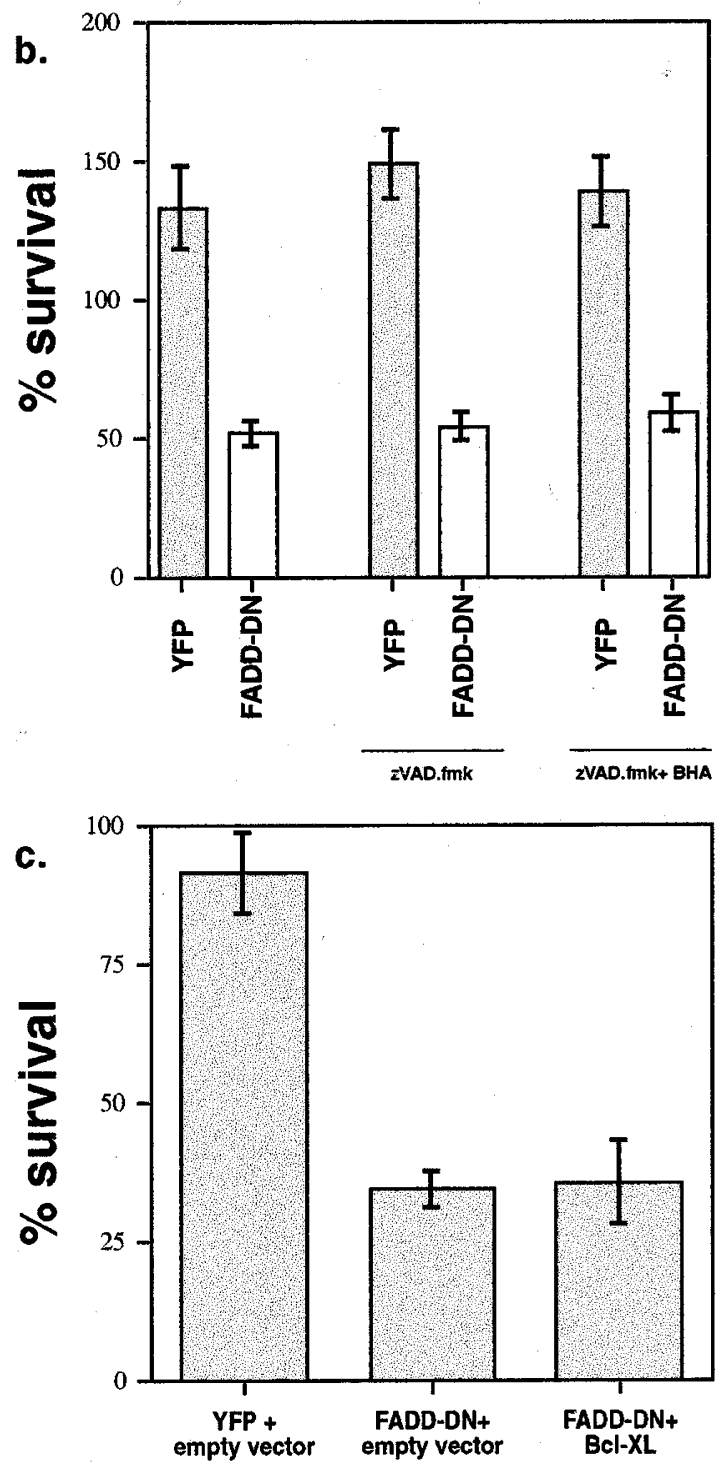

FADD is required to transduce apoptotic signals from activated death receptors and it is therefore surprising that an inhibitor of FADD should actually induce apoptosis. There are other examples where an apoptosis inhibitor can have the opposite effect. The c-FLIP protein, an endogenous inhibitor of Fas signaling that binds to the death effector domain of FADD to prevent caspase 8 activation can also induce apoptosis upon overexpression. ${ }^{29}$ While such observations with an inhibitor of Fas signaling inducing apoptosis are reminiscent of our results, an important difference indicates that the mechanism in our case is distinct from that of c-FLIP. c-FLIP can induce apoptosis in tumor-derived cell lines such as Hela cells ${ }^{30}$ that are not killed by FADD-DN. Because we only observe FADD-DN-induced death in a specific, normal cell type, our observations indicate a particular role for FADD in normal prostate epithelial cells that is lost in tumor cells. This role involves regulation of caspases but this is via a mechanism that is distinct from FADD's well-characterized recruitment and aggregation of caspase 8 via the death effector domain that is important during death receptor-induced apoptosis. ${ }^{3}$

Despite the fact that caspases were selectively activated in FADD-DN-expressing normal prostate epithelial cells, caspase inhibitors did not efficiently block FADD-DNinduced cell death. These data suggest that caspaseindependent death pathways, or caspases that are not inhibited by zVAD.fmk and p35 can also be activated by FADD-DN in normal prostate cells. Several apoptosis regulators that activate caspases can also kill cells via caspase-independent mechanisms. ${ }^{31}$ Examples include overexpressed Bax and Bak, two pro-apoptotic Bcl-2 family members, ${ }^{32,33}$ oncogenes such as $\mathrm{E} 1 \mathrm{a}$ and $\mathrm{Myc}^{33}$ and $\mathrm{PML}^{34}$. These examples were identified in transformed and/or immortalized cell lines and differ from our observations in that there is no evidence that these responses are different in tumor cells and normal cells. Our data suggest that both caspase activation and caspase-independent pathways contributing to FADD-DN-induced killing are cell type-specific and defective in prostate tumor cells. It has been suggested that caspase-independent death may involve mitochondrial defects leading to high levels of reactive oxygen species $^{35}$ or release of mitochondrial proteins such as apoptosis inducing factor (AIF). ${ }^{36}$ Release of AIF can be prevented by anti-apoptotic Bcl-2 proteins. ${ }^{36}$ Because overexpressed $\mathrm{BCl}-\mathrm{x}_{\mathrm{L}}$ did not prevent FADD-DN-induced death (Figure $5 \mathrm{c}$ ), our results are not consistent with this mechanism. After this manuscript was submitted, necrotic cell death associated with FADD was

Figure 5 Caspase inhibitors, antioxidants and $\mathrm{Bcl}-\mathrm{x}_{\mathrm{L}}$ do not efficiently prevent FADD-DN-induced apoptosis. (a) Normal prostate epithelial cells were injected with control or FADD-DN expression vectors in the presence or absence of $150 \mu \mathrm{M}$ zVAD.fmk and cell survival was determined. The caspase inhibitor caused only a very small increase in survival indicating that caspaseindependent pathways contribute to FADD-DN-induced death. Data shown is mean per cent survival + S.E.M. from five experiments. (b) Cells were injected with the YFP control or YFP-FADD-DN in the presence of ZVAD.fmk or zVAD.fmk plus $100 \mu \mathrm{M}$ BHA. The inhibitors did not prevent FADD-DN-induced death. (c) Normal prostate cells were injected with control vectors, FADD-DN or FADD-DN plus a Bcl- $x_{L}$ expression plasmid and cell survival was determined. Bcl- $x_{L}$ co-expression did not prevent FADD-DN-induced death 
a.

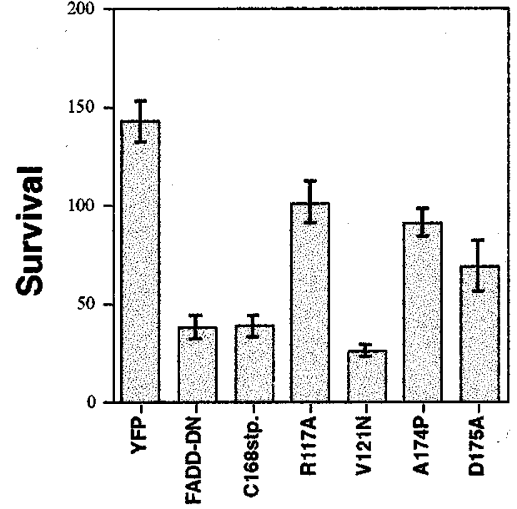

b.

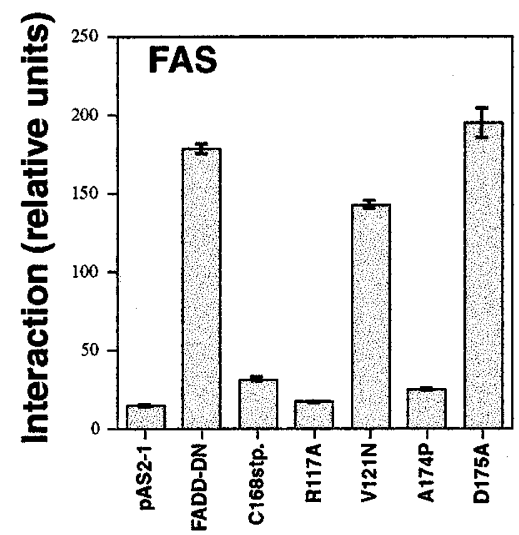

c.

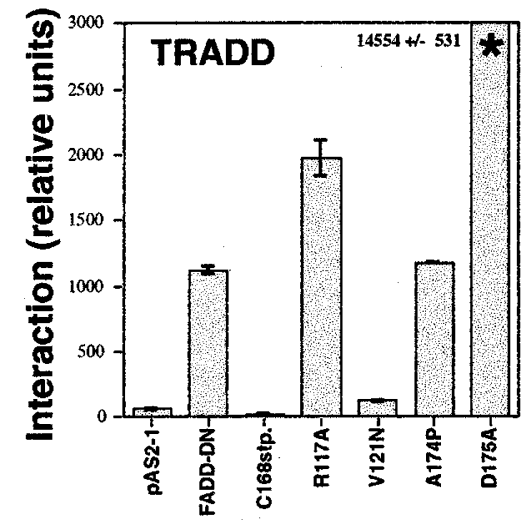

d.

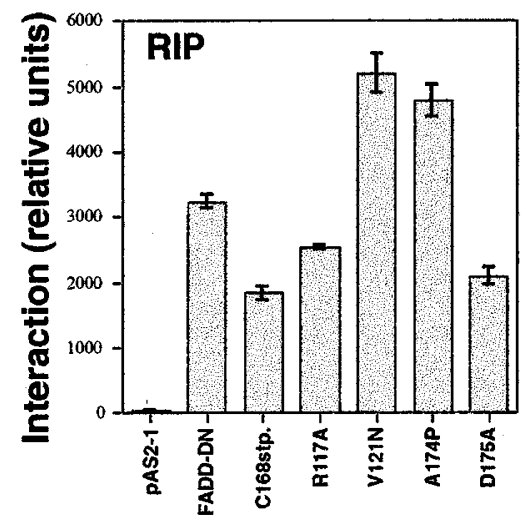

\section{Summary Table:}

e.

\begin{tabular}{|l|l|l|l|l|l|l|l|}
\hline & pAS2-1 & FADD-DN & C168stp. & R117A & V121N & A174P & D175A \\
\hline FAS-DD & - & ++++ & - & - & +++ & - & ++++ \\
\hline TRADD & & ++++ & - & +++++++ & - & ++++ & $+++++\times(\times 10)$ \\
\hline RIP & - & ++++ & ++ & +++ & ++++++ & ++++++ & +++ \\
\hline death & -- (YFP) & ++++ & ++++ & - & ++++ & - & + \\
\hline
\end{tabular}

Figure 6 FADD-DN-induced apoptosis is not associated with interaction with Fas, TRADD or RIP. (a) Cell survival after injection of FADD-DN or the indicated mutants was determined indicating that some mutants (C168 stop and V121N) were equally potent as the wild-type FADD-DN at inducing apoptosis, while the other mutants were less able to induce apoptosis. The data represents mean survival \pm S.E.M. from between five and ten separate preparations of normal human prostate epithelial cells for each mutant. (b-d) Directed two-hybrids were performed between the various mutants and Fas, TRADD or RIP normalized to that of the wild-type FADD-DN protein. (e) Summarizes the ability of each mutant to induce prostate cell apoptosis and interact with Fas, TRADD and RIP

reported by two groups. Matsumura et al. ${ }^{37}$ demonstrated that the FADD DED (but not the death domain) could kill caspase 8 deficient cells via a necrotic pathway that was associated with loss of mitochondrial membrane potential and the generation of reactive oxygen species. Boone et al. ${ }^{27}$ showed that FADD-DN could kill L929sA fibrosarcoma cells in a manner that was not inhibited by zVAD.fmk or CrmA, an efficient inhibitor of caspase 8. These authors showed that the death domain from TNFR1 caused a similar death that was inhibited by the antioxidant BHA. However, they did not test whether antioxidants prevented the FADD-DN-induced death in L929sA cells. We were unable to demonstrate any protection against FADD-DNinduced death using BHA along with zVAD.fmk (Figure $5 b$ ). These results indicate that the prostate cell death is different from these necrotic responses. Recently, a new form of programmed cell death termed paraptosis was described. ${ }^{38}$ This cell death required new protein synthesis and was unaffected by caspase inhibitors or $\mathrm{BCl}-\mathrm{x}_{\mathrm{L}}$. We do not know whether FADD-DN-induced death of normal prostate cells requires new protein synthesis because protein synthesis inhibitors are toxic to the primary prostate cells over the time courses that we have used. However, as reagents that are specific for paraptosis become available, it will be interesting to test whether FADD-DN can activate this mechanism in normal epithelial cells.

Normal cells require continual survival signals to resist apoptosis. ${ }^{39}$ Therefore, one hypothesis is that FADD-DN interferes with a signaling pathway that is required for prostate epithelial cell survival and that this pathway is bypassed in prostate cancer cells. FADD-DN consists only of a protein interaction domain and blocks death receptor- 
induced apoptosis because of its ability to interact with the receptor and disrupt the DISC. One question therefore is whether a FADD-dependent survival pathway whose inhibition leads to prostate cell apoptosis also involves signaling via the death receptors. Our experiments (Figure 6) using mutants that differ in their ability to kill cells and to interact with Fas and two other components of death receptor DISCs began to address this question. There was no correlation between Fas, TRADD and RIP binding and the ability to induce prostate cell apoptosis suggesting that FADD-DN is not interfering with death receptor signaling to cause prostate cell death.

There are several other indications of different roles for FADD in addition to the propagation of apoptosis signals from death receptors. For example, although death receptor knockouts are viable, FADD knockout mice die before birth showing defects in heart development, ${ }^{40}$ indicating a role in development that is distinct from death receptor-induced apoptosis signaling. In addition, FADDDN expression sensitizes NIH3T3 cells to necrotic death in response to $\mathrm{TNF}^{41,42}$ and impairs calcium mobilization. ${ }^{43}$ FADD-DN expression can also cause defects in $T$ cell proliferation ${ }^{43-46}$ indicating a role for FADD in cell growth as well as death. In some cases, FADD's function in growth regulation may involve c-FLIP, which when bound to the FADD death effector domain at the Fas receptor can activate the ERK and NF- $\kappa$ B pathways. ${ }^{47}$ In principal, this activity could be involved in the prostate cell apoptosis that we see. If c-FLIP and thus FADD-dependent signals via these pathways were required for epithelial cell survival, FADD-DN expression might inhibit the signals leading to prostate cell apoptosis. However, we think that this scenario is unlikely for two reasons. First, the activation of ERK and NF $\kappa \mathrm{B}$ pathways by c-FLIP occurs in response to Fas stimulation ${ }^{47}$ whereas our data indicates that FADDDN binding to Fas is not required for prostate cell apoptosis. Second, we cannot mimic the pro-apoptotic effect of FADD-DN in prostate epithelial cells when we inhibited these pathways in other ways by overexpressing $\mathrm{I}_{\kappa} \mathrm{B}$ or MAP kinase phosphatases or treating with MEK inhibitors (data not shown). We suggest that the prostate epithelia-specific functions for FADD indicated by our results represent another distinct function for this adaptor protein. It is possible that some of the other FADD functions that have been identified are also independent of death receptor binding.

In conclusion, we have shown that in prostate epithelial cells a dominant-negative inhibitor of FADD can induce caspase activation and apoptosis. Thus, the FADD death domain participates in a cell type-specific pathway that is distinct from its role in mediating apoptotic signals from death receptors such as Fas. Of most potential importance, this novel pathway is apparently non-functional in both primary prostate tumor cells and prostate tumor cell lines suggesting that it may be important in regulating prostate carcinogenesis. Identification of proteins that are responsible for FADD-DN induced death in prostate epithelial cells should allow us to characterize the relevant signaling pathway and to understand why it is not active in nonepithelial cells and tumor-derived epithelial cells. Compo- nents of the pathway could be attractive targets for therapeutics to reactivate the pathway and kill prostate tumor cells.

\section{Materials and Methods}

\section{Cell culture and microinjection}

Primary human prostate epithelial cells were cultured from discarded tissue pieces obtained during surgery. Tissue pieces from regions of the gland that were distinct from tumor foci and contained only morphologically normal tissue (normal cells), or tissue pieces that were determined by the attending surgical pathologist to contain tumor (primary tumor cells) were minced into small pieces and processed as previously described. ${ }^{48}$ The cells were plated onto collagen-coated etched coverslips and maintained in PrEBM media with PrEGM bullet kit media (Clonetics BioWhittaker, La Jolla, CA, USA). For comparison between prostate stromal and epithelial cells, normal primary prostate epithelial, fibroblast or smooth muscle cells were obtained from Clonetics and maintained as recommended by the supplier. Normal human skin fibroblasts and prostate cancer cell lines were maintained in DMEM. Expression plasmids at $250 \mathrm{ng} / \mu \mathrm{l}$ were injected into the nucleus of cells using an Eppendorf microinjector. Where indicated, cells were incubated in the presence of the caspase inhibitor zVAD.fmk $(150-500 \mu \mathrm{M})$ and $100 \mu \mathrm{M}$ butylated hydroxyanisole (Sigma, St. Louis, MO, USA) or the corresponding volume of DMSO or ethanol as a control. The YFP-FADD-DN expression plasmid was constructed in the YFP C1 expression vector from Clontech (Palo Alto, CA, USA) using a FADD-DN fragment provided by Harald Wajent. ${ }^{13}$ Point mutants were constructed by site-directed mutagenesis using the Quickchange kit (Stratagene, La Jolla, CA, USA). The DAP kinase death domain was amplified from a brain CDNA library and inserted into the YFP expression vector to produce the YFP-DAPK-DD expression plasmid.

\section{Apoptosis assays}

To score cell death, the fluorescent cells were counted $2 \mathrm{~h}$ after injection and the per cent survival was determined after overnight incubation. Quantitative data shown in the histograms represents the mean per cent survival ( \pm S.E.M.) from separate experiments of $50-$ 150 cells per sample using different preparations of cells and plasmids. Where indicated statistical significance between control and FADD-DN expressing cells was determined using a $t$-test. Annexin $V$ staining was performed on unfixed non-permeabilized cells as recommended by the manufacturer (Roche). Caspasecleaved cytokeratin 18 was detected using the M30 antibody (Roche) according to the manufacturers instructions.

\section{Fluorescence resonance energy transfer assay for caspase activation}

We previously described a single cell caspase assay using a YFP-BFP fusion protein that undergoes FRET when intact but not when cleaved by DEVDases. This assay allows continuous measurement of caspase activity in individual cells based on measurement of the ratio of yellow to blue fluorescence after excitation with UV radiation in a fluorescence microscope. ${ }^{26}$ For microscopy, cells were maintained in an environmental chamber at $37^{\circ} \mathrm{C}$ on a Zeiss axiovert microscope at $5 \% \mathrm{CO}_{2}$. Phase and fluorescence images were captured at $30 \mathrm{~min}$ intervals throughout the experiment. Yellow/blue ratio images were 
created from the primary data using the ratio module of the OpenLab (Improvision, Warwick, UK) software running on a Macintosh G3 computer. Quantitative data was obtained for individual cells by creating a region of interest around the cell and then measuring the number of pixels of the greyscale images using the OpenLab software. For each cell, the average yellow and blue intensity for its defined area was determined and the average background intensity (determined from an equivalent adjacent area with no cell present) at each time point was subtracted. The yellow/blue ratio was then determined by dividing the mean intensity of the yellow picture divided by the mean intensity for the blue picture for each time point. To compare control and FADD-DN-injected cells, the percentage change in the yellow/blue ratio $8-10 \mathrm{~h}$ after injection was calculated for individual cells that were injected either with control vector, or an untagged FADD-DN vector plus the fusion protein expression plasmid. The mean percentage changes were calculated for groups of control- and FADD-DN-injected cells. Statistical significance between the means was determined using a $t$-test.

\section{Two-hybrid interactions}

To measure interactions between FADD-DN mutants and either Fas, RIP or TRADD, directed two-hybrids were performed. FADD-DN and the various mutants were cloned into the pAS2-1 vector (Clontech). Fas (cytoplasmic domain, amino acids 177-335), RIP (full-length) and TRADD (full-length) cDNAs were cloned into pACT3-1. The plasmids were co-transformed into the yeast strain Y190 (Clontech), which contains a Gal4-regulated beta-galactosidase gene. Five individual clones were picked, pooled and grown up for each transformant. Betagalactosidase activity was determined in triplicate using a chemiluminescent assay kit (Tropix, Bedford, MA, USA) to assess the strength of interaction between each of the mutants and each target protein. The beta-galactosidase activities for each mutant were normalized so that interaction with the wild-type FADD-DN molecule was $100 \%$.

\section{Acknowledgements}

We thank Gerard Evan, Scott Cramer, David Virshup and Don Ayer for discussions and comments on the manuscript. We are grateful to Harald Wajent who provided a FADD-DN cDNA. Prostate tissue was obtained through the Huntsman Cancer Institute Tissue Procurement Core Facility. This work was partially supported by the Huntsman Cancer Foundation, Wake Forest University School of Medicine and the North Carolina Biotechnology Center (A Thorburn) and NIH grant R01-46269 (AR Brothman).

\section{References}

1. Greenlee RT, Murray T, Bolden S and Wingo PA (2000) Cancer statistics, 2000. CA Cancer. J. Clin. 50: 7-33

2. Hanahan D and Weinberg RA (2000) The hallmarks of cancer. Cell. 100:57-70

3. Ashkenazi A and Dixit VM (1998) Death receptors: signaling and modulation. Science 281: 1305-1308

4. Rokhlin O, Bishop GA, Hostager BS,Waldschmidt TJ, Sidorenko SP, Pavloff N, Kiefer MC, Umansky SR, GLover RA and Cohen MB. (1997) Fas-mediated apoptosis in human prostatic carcinoma cell lines. Cancer Res. 57: 1758-1768

5. Rokhlin OW, Glover RA and Cohen MB (1998) Fas-mediated apoptosis in human prostatic carcinoma cell lines occurs via activation of caspase-8 and caspase-7. Cancer Res. 58: 5870-5875

6. Hedlund TE, Duke RC, Schleicher MS and Miller GJ (1998) Fas-mediated apoptosis in seven human prostate cancer cell lines: correlation with tumor stage. Prostate 36: $92-101$
7. Suzuki A, Matsuzawa A and Iguchi T (1996) Down regulation of $\mathrm{Bcl}-2$ is the first step on Fas-mediated apoptosis of male reproductive tract. Oncogene 13: 31 37

8. Powell WC, Fingleton B, Wilson CL, Boothby M and Matrisian LM (1999) The metalloproteinase matrilysin proteolytically generates active soluble fas ligand and potentiates epithelial cell apoptosis. Curr. Biol. 9: 1441-1447

9. Chinnaiyan AM, O'Rourke K, Tewari M and Dixit VM (1995) FADD, a novel death domain-containing protein, interacts with the death domain of Fas and initiates apoptosis. Cell 81: 505-512

10. Chinnaiyan AM, Tepper CG, Seldin MF, O'Rourke K, Kischkel FC, Hellbardt S, Krammer PH, Peter MA and Dixit VM. (1996) FADD/MORT1 is a common mediator of CD95 (Fas/APO-1) and tumor necrosis factor receptor-induced apoptosis. J. Biol. Chem. 271: 4961-4965

11. Hsu H, Shu H-B, Pan M-G and Goeddel DV (1996) TRADD-TRAF2 and TRADDFADD interactions define two distinct TNF receptor 1 signal transduction pathways. Cell 84: 299-308

12. Hueber A-O, Zornig M, Lyon D, Suda T, Nagata S and Evan GI (1997) Requirement for the CD95 receptor-ligand pathway in c-Myc-induced apoptosis. Science 278: 1305-1309

13. Wajant H, Johannes F-J, Haas E, Siemienski K, Schwenzer R, Schubert G, Weiss T, Grell M and Scheurich P. (1998) Dominant-negative FADD inhibits THFR60-, Fas/Apo1- and TRAIL-R/Apo2-mediated cell death but not gene induction. Current Biology 8: 113-116

14. Walczak H, Degli-Esposti MA, Johnson RS, Smolak PJ, Waugh JY, Boiani N, Timour MS, Gerhart MJ, Schooley KA, Smith CA, Goodwin RG and Rauch CT. (1997) TRAIL-R2: a novel apoptosis-mediating receptor for TRAIL. EMBO J. 16: $5386-5397$

15. Yang X, Kosravi-Far R, Chang HY and Baltimore D (1997) Daxx, a novel fasbinding protein that activates JNK and apoptosis. Cell 89: 1067-1076

16. Choi KB, Wong F, Harlan JM, Chaudhary PM, Hood L and Karsan A (1998) Lipopolysaccharide mediates endothelial apoptosis by a FADD-dependent pathway. J. Biol. Chem. 273: 20185-20188

17. Micheau O, Solary E, Hammann A and Dimanche-Boitrel MT (1999) Fas ligandindependent, FADD-mediated activation of the fas death pathway by anticancer drugs. J. Biol. Chem. 274: 7987-7992

18. Tang D, Lahti JM, Grenet J and Kidd VJ (1999) Cycloheximide-induced T-cell Death Is Mediated by a Fas-associated Death Domain-dependent Mechanism. J. Biol. Chem. 274: 7245-7252

19. Rytomaa M, Martins LM and Downward J (1999) Involvement of FADD and caspase-8 signalling in detachment-induced apoptosis. Curr. Biol. 9: 10431046

20. Frisch SM (1999) Evidence for a function of death-receptor-related, deathdomain- containing proteins in anoikis. Curr. Biol. 9: 1047-1049

21. Sanchez I, Xu CJ, Juo P, Kakizaka A, Blenis J and Yuan J (1999) Caspase-8 is required for cell death induced by expanded polyglutamine repeats. Neuron. 22 : $623-633$

22. Alberts AS, Frost JA and Thorburn AM (1993) Rapid transcription assay for the expression of two distinct reporter genes by microinjection. DNA and Cell Biology 12: $935-943$

23. Cohen O, Inbal B, Kissil JL, Raveh T, Berissi H, Spivak-Kroizaman T, Feinstein E and Kimchi A. (1999) DAP-kinase participates in TNF-alpha- and Fas-induced apoptosis and its function requires the death domain. J. Cell. Biol. 146: 141-148

24. Horoszewicz JS, Leong SS, Kawinski E, Karr JP, Rosenthal H, Chu TM, Mirand EA and Murphy GP. (1983) LNCaP model of human prostatic carcinoma. Cancer Research 43: 1809-1818

25. Brothman AR, Lesho LJ, Somers KK, Wright GL and Merchant DJ (1989) Phenotypic and cytogenetic characterization of a cell line derived from primary prostatic carcinoma. Int. J. of Cancer 44: 898-903

26. Morgan MJ and Thorburn A (2001) Measurement of caspase activity in individual cells reveals differences in the kinetics of caspase activation between cells. Cell Death \& Differ. 8: $38-43$

27. Boone E, Vanden Berghe T, Van Loo G, De Wilde G, De Wael N, Vercammen D, Fiers W, Haegeman G and Vandenbeele P. (2000) Structure/Function analysis of 555 tumornecrosis factor receptor and fas-associated death domain. Effect on necrosis in L929sA cells. J. Biol. Chem. 275: 37596-37603

28. Zhang SQ, Kovalenko A, Cantarella G and Wallach D (2000) Recruitment of the IKK signalosome to the p55 TNF receptor: RIP and A20 bind to NEMO (IKKgamma) upon receptor stimulation. Immunity 12: 301-311 
29. Tschopp J, Irmler M and Thome M (1998) Inhibition of fas death signals by FLIPs. Curr. Opin. Immunol. 10: 552-558

30. Shu HB, Halpin DR and Goeddel DV (1997) Casper is a FADD- and caspaserelated inducer of apoptosis. Immunity 6: 751-763

31. Borner C and Monney L (1999) Apoptosis without caspases: an inefficient molecular guillotine? Cell Death Differ. 6: 497-507

32. Xiang J, Chao DT and Korsmeyer SJ (1996) BAX-induced cell death may not require interleukin $1 B$-converting enzyme-like proteases. Proc. Natl. Acad. Sci. USA. 93: $14559-14563$

33. McCarthy NJ, Whyte MK, Gilbert CS and Evan GI (1997) Inhibition of Ced-3/ICErelated proteases does not prevent cell death induced by oncogenes, DNA damage, or the Bcl-2 homologue Bak. J. Cell. Biol. 136: 215-227

34. Quignon F, De Bels F, Koken M, Feunteun J, Ameisen JC and de The H (1998) PMLinduces a novel caspase-independent death process. Nat. Genet. 20:259 265

35. Green DR and Reed JC (1998) Mitochondria and apoptosis. Science 281: $1309-1312$

36. Susin SA, Lorenzo HK, Zamzami N, Marzo I, Snow BE, Brothers GM, Mangion J, Jacotot E, Costantini P, Loeffler M, Larochette N, Goodlett DR, Aebersold R, Siderovski DP, Penninger JM and Kroemer G. (1999) Molecular characterization of mitochondrial apoptosis-inducing factor. Nature 397: 441-446

37. Matsumura H, Shimizu $Y$, Ohsawa $Y$, Kawahara A, Uchiyama $Y$ and Nagata $S$ (2000) Necrotic Death Pathway in Fas Receptor Signaling. J. Cell. Biol. 151: $1247-1256$

38. Sperandio S, de Belle I and Bredesen DE (2000) An alternative, nonapoptotic form of programmed cell death. Proc. Natl. Acad. Sci. USA. 97: 14376-14381

39. Raff MC (1992) Social controls on cell survival and cell death. Nature 356: 397400

40. Yeh W-C, Pompa JLDL, McCurrach ME, Shu H-B, Elia AJ, Shahinan A, Ng M, Wakeham A, Khoo W, Mitchell K, El-Diery WS, Lowe SW, Goeddel DV and Mak TW. (1998) FADD: essential for embryo development and signaling from some, but not all, inducers of apoptosis. Science 279: 1954-1958
41. Khwaja A and Tatton $L$ (1999) Resistance to the cytotoxic effects of tumor necrosis factor alpha can be overcome by inhibition of a FADD/Caspasedependent signaling pathway. J. Biol. Chem. 274: 36817-36823

42. Luschen S, Ussat S, Scherer G, Kabelitz D and Adam-Klages S (2000) Sensitization to death receptor cytotoxicity by inhibition of fas-associated death domain protein (FADD)/caspase signaling. Requirement of cell cycle progression. J. Biol. Chem. 275: 24670-24678

43. Hueber AO, Zornig M, Bernard AM, Chautan M and Evan G (2000) A dominant negative Fas-associated death domain protein mutant inhibits proliferation and leads to impaired calcium mobilization in both T-cells and fibroblasts. J. Biol. Chem. 275: 10453-10462

44. Zornig M, Hueber AO and Evan G (1998) p53-dependent impairment of T-cell proliferation in FADD dominant- negative transgenic mice. Curr. Biol. 8: 467470

45. Newton K, Harris AW, Bath ML, Smith KGC and Strasser A (1998) A dominan interfering mutant of FADD/MORT1 enhances deletion of autoreactive thymocytes and inhibits proliferation of mature T lymphocytes. EMBO J. 17 $706-718$

46. Walsh CM, Wen BG, Chinnaiyan AM, O'Rourke K, Dixit VM and Hedrick SM (1998) A role for FADD in T cell activation and development. Immunity 8: 439449

47. Kataoka T, Budd RC, HollerN, Thome M, Martinon F, Irmler M, Burns K, Hahne M, Kennedy N, Kovacsovics M and Tschopp J. (2000) The caspase-8 inhibitor FLIP promotes activation of NF-kappaB and erk signaling pathways. Curr. Biol. 10: 640-648

48. Brothman AR, Peehl DM, Patel AM and McNeal JE (1990) Frequency and pattern of karyotypic abnormalities in human prostate cancer. Cancer Res. 50: 3795 3803 